\title{
Die Entwicklung der jamaikanischen Arbeiterbank in den siebzi- ger Jahren
}

\author{
von Dieter Semmelroth
}

\section{Einleitung}

Schon frühzeitig haben sich die Gewerkschaften in den Industrieländern für Selbsthilfeaktivitäten der abhängig Beschäftigten ausgesprochen. In diesem Zusammenhang ist auch die Entscheidung der Arbeiterorganisationen zu s,ehen, Spar- und Darlehensvereine der Arbeiterschaft zu unterstützen und später selbst Bankinstitute zu gründen. ${ }^{1}$ Den meisten dieser Banken waren allerdings keine oder allenfalls beschränkte wirtschaftliche Erfolge beschieden. Ausnahmen bilden lediglich die israelische Bank Hapoalim ${ }^{2}$ sowie die bundesdeutsche Bank für Gemeinwirtschaft, ${ }^{3}$ die zu den führenden Banken ihrer Länder zählen. Inzwischen wurden auch in Ländern der Dritten Welt eine Reihe von Arbeiterund Gewerkschaftsbanken gegründet. In den letzten fünfzehn Jahren entstanden in Lateinamerika und der Karibik ${ }^{4}$ elf und in Malaysia eine Arbeiterbank.

Im folgenden soll eine dieser Neugründungen näher dargestellt und analysiert werden. Es handelt 'sich dabei um die 1973 auf der Karibikinsel Jamaika gegründete 'Workers' Savings and Loan Bankı. Es werden vor allem die Fragen untersucht, warum es zur Gründung der Arbeiterbank kam, wie die Bank aufgebaut ist, wie sie sich geschäftlich entwickelt hat und welchen Problemen sie sich gegenübersieht.

\section{Ausgangslage}

Im Jahre 1962 wurde Jamaika (Fläche ca. 11000 qkm; ca. 2 Mio. Einwohner) nach über dreihundertjähriger Kolonialherrschaft unabhängig. Die Briten hinterließen eine

1 Zur historischen Entwicklung in Deutschland vgl. von Loesch, A., Die deutschen Arbeiterbanken in den zwanziger Jahren, Frankfurt/M. 1974; zur Entwicklung in den USA vgl. von Loesch, A., Die nordamerikanischen Arbeiterbanken in den zwanziger Jahren, Frankfurt/M. 1972.

2 Vgl. Lewinsky, A., El Banco de los Trabajadores de Israel: Bank Hapoalim, B. M., Tel Aviv 1968.

3 Vgl. von Loesch, A., Die Bank für Gemeinwirtschaft, Frankfurt/M. ${ }^{2} 1977$.

4 Arbeiterbanken entstanden in Puerto Rico (1961), Guatemala (1965), Honduras (1966), Argentinien (1968), Costa Rica (1969), Guyana (1970), Trinidad und Tobago (1971), Jamaika (1973), Dominikanische Republik (1974), Kolumbien (1974), Paraguay (1975) und Mexiko (1977). Uberblicke über die Entwicklung dieser Banken geben Mitchell, N., A Comparative Study of Workers' Banks - Some Aspects of their evelopment, Role, Policies and Perspectives, Organization of American States, Capital Market Program, Washington 1974; Lewerenz, J., Die Arbeiterbanken in Lateinamerika und in der Karibik, Frankfurt/M. 1976; CEDAL (Hg.), La Banca de Interés Social en América Latina - Colleccion y Documentos, San José, C. R. 1977 und American Insitut of Free Labor Development (Hg.), Report of the Meeting of Latin American and Caribbean Workers' Banks, Washington 1979. 
krisenanfällige Plantagenwirtschaft mit nur einem kleinen modernen Sektor, der allerdings kaum in der Lage war, einen wesentlichen Beitrag zur Linderung der hohen Dauerarbeitslosigkeit zu leisten. Auch in den folgenden Jahren blieben die zentralen Bereiche der jamaikanischen Wirtschaft fest im Griff ausländischer Investoren. ${ }^{5}$ Selbst eine Dekade nach Erlangung der Unabhängigkeit waren noch alle acht auf der Karibikinsel tätigen Geschäftsbanken im Besitz nordamerikanischer und britischer Mutterunternehmen. Lediglich zwei Unternehmen hatten damit begonnen, Minderheitsbeteiligungen an jamaikanische Interessenten anzugeben.

$\mathrm{Zu}$ Beginn der siebziger Jahre geriet die Geschäftspolitik dieser von ausländischen Interessen beherrschten Banken in die öffentliche Kritik. Bemängelt wurde vor allem, daß die Institute ihre finanziellen Ressourcen zu wenig für die nationale Entwicklung Jamaikas einsetzten. ${ }^{6}$ Kritisiert wurde weiterhin, daß sich die Geschäftsbanken, die ca. $80 \%$ aller Spareinlagen verwalteten, in ihrem Kreditgeschäft nur auf wenige Branchen und Geschäftsarten konzentrierten. Bevorzugten Zugang zu Bankkrediten hatten hauptsächlich kapitalintensiv wirtschaftende Unternehmen des modernen Sektors sowie große Außenhandelsfirmen. Demgegenüber waren von Jamaikanern betriebene Klein- und Mittelbetriebe, die für den heimischen Markt arbeitsintensiv produzierende Landwirtschaft und der informelle Sektor kaum als Kreditnehmer vertreten. Nach einer Analyse der Kreditpolitik der Geschäftsbanken kam ein jamaikanischer Wirtschaftswissenschaftler zu dem Ergebnis, daß". . . commercial Bank credit in Jamaica has not been particularly responsive to the domestic needs, and has operated in the interest of the foreign capital. " 7 Diese Kritik an der Geschäftspolitik der Banken machten sich auch die jamaikanischen Gewerkschaften zueigen. In ihren Reihen entstand die Idee, eine jamaikanische Bank unter Beteiligung der Gewerkschaften, der Genossenschaften und des Staates zu gründen. Erstmals wurde dieser Vorschlag 1971 von Thossy Kelly, dem damaligen Vorsitzenden der National Workers Union (NWU), zur Diskussion gestellt. ${ }^{8}$ Der aus der Gewerkschaftsbewegung hervorgegangene Premierminister, Michael Manley, nahm die Anregung auf und bekundete das Interesse seiner Regierung an der Gründung einer solchen Bank. Als mögliche Aufgabenfelder nannte er:

- die Entwicklung und Einführung von besonders auf die Bedürfnisse der Arbeiter und Personen mit niedrigen Einkommen zugeschnittenen Sparprogrammen;

- die Bereitstellung von Mitteln für kurzfristige Kredite auf der Basis neuer Sicherheitsanforderungen und -normen;

- die Entwicklung von Wohnbaufinanzierungsprogrammen für Bezieher kleinerer und mittlerer Einkommen;

5 Vgl. Girvan, N., Foreign Capital and Economic Underdevelopment in Jamaica, Kingston 1971; Jefferson, O., The Post-War Economic Development of Jamaica, Kingston 1972.

6 Vgl. Kirton, C., Venner, D., Commercial Banking in Jamaica: A General Overview, Kingston o. J. (hekt.), S. 14 ff.; Tucker, P., Development of Workers Bank, Kingston 1977 (hekt.), S. $1 \mathrm{ff}$.

7 Kirton, C., Bank Lending and Economic Underdevelopment in Jamaica: Can an Indigeneous Bank Solve the Problem?, in: Social and Economic Studies, 3 (1977), S. 314.

$8 \mathrm{Vgl}$. Gayle, A., The Struggles of the Jamaican Workers, Kingston 1980 (hekt.), S. 64. 
- das Angebot von Dienstleistungen im Bereich der Vermögensverwaltung sowohl für im Lande als auch in Ubersee lebende Jamaikaner;'

- die Bereitstellung von Beratungsdiensten in Finanzierungs- und Beratungsfragen für kleinindustrielle Unternehmen und Genossenschaften. ${ }^{10}$

Ebenso sprach sich der Finanzminister für die Gründung einer Arbeiterbank aus. Er betonte allerdings, daß die Bank trotz aller reformpolitischer Absichten auf keinen Fall ihre ökonomische Lebensfähigkeit auf das Spiel setzen dürfte. "As an operational yardstick the Finance Minister stated that the Bank should operate as a commercially viable institution in such a manner as to make a reasonable profit consistent with the broad policy objective of providing better and wider banking services to workers and Jamaican owned enterprises. "11

Der Premierminister ernannte im Mai 1972 eine Arbeitsgruppe, ${ }^{12}$ die konkrete Vorstellungen für den Aufbau einer Arbeiterbank erarbeiten sollte. Die Gruppe studierte sorgfältig die Entwicklung und Organisation europäischer, israelischer und lateinamerikanischer Arbeiterbanken. Insbesondere wurden die Erfahrungen der israelischen und der deutschen Arbeiterbank analysiert und auf ihre Verwendbarkeit für die jamaikanischen Verhältnisse untersucht.

Die Arbeitsgruppe kam zu dem Ergebnis, daß die Bank die größte Wirkung erzielen könne, wenn sie ihren Kundenkreis nicht nur auf Arbeiter und Bezieher kleinerer Einkommen beschränke sondern alle sozialen Schichten anspreche. Weiterhin empfahl die Gruppe, daß

- Profitmaximierung nicht Hauptziel der Arbeiterbank sein sollte, wenngleich die Bank doch einen angemessenen Gewinn für ihre Aktionäre erwirtschaften sollte;

- die Geschäftspolitik der Bank einen Beitrag zur Steigerung des Lebensstandards der Arbeiterschaft und der 'kleinen Leuter leisten sollte;

- die Arbeiterbank die bestehende Postsparkasse mit ihrem Agenturnetz in allen Postdienststellen übernehmen solle. ${ }^{13}$

Diese Vorschläge sowie detaillierte Angaben zur Struktur und Organisation der zu gründenden Bank fanden Eingang in ein spezielles Arbeiterbankgesetz, das 1973 verabschiedet wurde. ${ }^{14}$ Neben den schon angeführten Zielsetzungen sieht das Gesetz vor, daß die Bank vor allem arbeitsintensive Vorhaben finanzieren sowie das Wachstum der jamaikanischen Volkswirtschaft durch die Förderung der Klein- und Mittelindustrie sowie der Genossenschaften unterstützen sollte. ${ }^{15}$

9 Ca. 1 Mio. Jamaikaner leben in Ubersee (vor allen in den USA, Großbritannien und Kanada).

10 Vgl. Tucker, P., a.a.O., S. 4.

11 Tucker, P., a.a.O., S. 5.

12 Der Arbeitsgruppe gehörten Vertreter der Gewerkschaften, der Genossenschaften, der Nationalbank sowie verschiedener Fachministerien an.

13 Vgl. Report of the Working Party to Establish a Workers Bank, Kingston 1972 (hekt.), S. 2 f.

14 The Workers Savings and Loan Bank Act, 1973 (Act 26 of 1973).

$15 \mathrm{Vgl}$. ebd., S. 4 f. 


\section{Aufbau und Organisation der Arbeiterbank}

Die jamaikanische Arbeiterbank, die den Namen 'Workers Savings and Loan Bank' (WSLB) erhielt, nahm am 1. Oktober 1973 ihre Geschäftstätigkeit auf. Von den übrigen Geschäftsbanken unterscheidet sie sich in der Zusammensetzung des Eigenkapitals, in der Zusammensetzung ihres Aufsichts- und Entscheidungsgremiums (Board of Directors) sowie in der Unternehmenszielsetzung.

Das authorisierte Kapital der in der Rechtsform einer Aktiengesellschaft gegründeten WSLB wurde auf J \$ 10 Mio. ${ }^{16}$ festgesetzt. Wie bei den anderen Arbeiterbanken in Lateinamerika wurde das Kapital durch die Ausgabe von Aktien verschiedener Klassen aufgebracht, die mit unterschiedlichen Stimmrechten versehen sind.$^{17}$ Durch diese Differenzierung sollte sichergestellt werden, daß der Bank sowohl genügend Eigenkapital zufließt als auch daß die Arbeiterorganisationen den bestimmenden Einfluß auf die Bankpolitik haben. Daher sieht das Arbeiterbankgesetz die Ausgabe von Aktien der Klassen A, B, C und D vor. Aktien der Klasse A sind für den Staat und parastaatliche Organisationen reserviert. Aktien der Klasse B können ausschließlich von Gewerkschaften erworben werden. Entsprechend der geringen Finanzkraft dieser Organisationen wurde das authorisierte Kapital für Klasse B Aktien auf J $\$ 400000$ festgesetzt. Während die Aktien der Klassen A und B nicht übertragbar sind, sind die Papiere der Klasse C frei handelbar. Sie sind für die Offentlichkeit einschließlich der Genossenschaften und Credit Unions bestimmt. Die Aktien der Klassen A, B, und C sind im Gegensatz zu denjenigen der Klasse D mit Stimmrechten versehen. Anteilpapiere der Klasse D können ausschließlich vom Staat erworben werden.

Der Nominalwert von 50 cents pro Aktie wurde bewußt niedrig gehalten, um eine breite Kapitalstreuung zu erreichen. Tatsächlich ist die WSLB mit über 10000 Aktionären die größte Publikumsgesellschaft der Karibikinsel. Als 1973 die Aktien auf den Markt kamen, waren sie innerhalb weniger Tage vergriffen. ${ }^{18}$ Der Appell an das Nationalgefühl der Jamaikaner und die Werbung der Gewerkschaften, die sich vor allem in der Gründungsphase für die Bank stark machten, zahlte sich aus. Der anfängliche Enthusiasmus wich jedoch bald einer Ernüchterung. Die Unzufriedenheit der Gewerkschaften, Genossenschaften und der Offentlichkeit mit der Geschäftspolitik und der Ertragslage der Bank führte dazu, daß sich diese Aktionäre mit weiteren Kapitalbeteiligungen zurückhielten. Um den Eigenkapitalbedarf der WSLB zu decken, mußte die Regierung ihren Anteil an Klasse D Aktien erheblich aufstocken. ${ }^{19}$ Dadurch ist inzwischen der Staat zu

$161 \mathrm{~J} \$=1,10 \mathrm{DM}$ (Stand 12/1980).

17 Die Ausgabe von Aktien unterschiedlicher Klassen geht auf das Vorbild der Arbeiterbank von Puerto Rico zurück. Die Organisation Amerikanischer Staaten (OAS) nahm die puertoricanische Arbeiterbankkonzeption auf und empfahl sie ihren Mitgliedstaaten zur Nachahmung. Zur Rolle der OAS bei der Verbreitung der Arbeiterbanken in Lateinamerika und der Karibik vgl. Mitchell, N., a.a.O., $1 \mathrm{ff}$.; Lewerenz, J., a.a.O., S. 7 ff.

$18 \mathrm{Vgl}$. Workers Savings and Loan Bank, Sunday Gleaner, 21. 10. 1973.

19 Zur Eigenkapitalstruktur vgl. Tab. 1, Anhang. 
über $80 \%$ am Kapital der Arbeiterbank beteiligt. Diese Eigenkapitalstruktur gibt Anlaß zur Sorge, daß der faktische Einfluß des Staates auf die Bank immer stärker wird. Das Arbeiterbankgesetz regelt die Zusammensetzung des dreizehnköpfigen Direktoriums der WSLB. ${ }^{20}$ Stimmrechtsregelungen sorgen dafür, daß die Arbeiter- und Selbsthilfeorganisationen unabhängig von ihrer Kapitalbeteiligung im Board of Directors vertreten sind. Drei Direktoren werden vom Finanzminister ernannt, fünf stellen die Gewerkschaften, drei sind Vertreter der Klasse C Aktionäre. Das Direktorium wird durch den Generalmanager und einen weiteren Vertreter des Managements vervollständigt. Von den übrigen auf Jamaika tätigen Geschäftsbanken unterscheidet sich die WSLB ferner in ihrer Unternehmenszielsetzung. Aufgrund ihrer programmatischen Aussagen will die Arbeiterbank sowohl im Wettbewerb bestehen und einen angemessenen Gewinn erwirtschaften als auch spezielle Programme durchführen und Projekte fördern, die einen hohen entwicklungspolitischen und sozialen Ertrag versprechen. Zur Realisierung dieser anspruchsvollen Zielsetzung will die WSLB in Geschäftsbereiche vorstoßen, die von der Konkurrenz bisher vernachlässigt worden sind. Insgesamt soll die geschäftliche Tätigkeit der Arbeiterbank Arbeitern und Unterprivilegierten zugute kommen, die traditionell vom Bankensystem nur wenig beachtet wurden.

Die Organisationsstruktur der WSLB basiert auf den funktionalen Beziehungen zwischen der in Kingston angesiedelten Zentrale und den städtischen und ländlichen Zweigstellen. Den Fachabteilungen der Zentrale obliegt die Aufgabe der Erstellung zentraler Dienstleistungen sowie der Úberwachung und Koordination der Zweigstellenaktivitäten. ${ }^{21}$ Die Zweigstellenpolitik der WSLB war von Anfang an auf Expansion ausgelegt. Wegen der ökonomischen Krise der siebziger Jahre ${ }^{22}$ konnte der Ausbau des Filialnetzes allerdings nicht im ursprünglich geplanten Umfang realisiert werden. Bis Ende 1980 arbeiteten in Kingston und den Provinzstädten 12 Zweigstellen. Darüber hinaus bestanden in rund 260 Postdienststellen WSLB Agenturen. Durch dieses umfassende Netz ist die Arbeiterbank auch in ländlichen Gebieten vertreten, wo sonst keine Geschäftsbanken tätig sind. Wie jedoch schon früher die von der WSLB übernommene Postsparkasse, so bietet die Arbeiterbank in ihren Agenturen nur Einlagemöglichkeiten. Zur Zeit stammen ca. $45 \%$ aller Depositen aus dem ländlichen Agenturnetz. ${ }^{23}$ Zeitweise wurden Úberlegungen angestellt, weitere Bankleistungen über die WSLB Agenturen laufen zu lassen. Dieses Vorhaben konnte jedoch wegen der ablehnenden Haltung der Postverwaltung nicht durchgeführt werden. Damit fließt im ländlichen Raum angespartes Kapital auch weiterhin in die Städte, ohne daß neue organisatorische Möglichkeiten für einen Rücktransfer geschaffen worden sind.

$20 \mathrm{Vgl}$. The Workers Savings and Loan Bank Act, S. $12 \mathrm{ff}$.

21 Vgl. Workers Savings and Loan Bank - Organizational Chart o. O., o. J., S. 2 f.

22 Zur wirtschaftlichen Entwicklung Jamaikas in der letzten Dekade vgl. EPICA Task Force (Hg.), Jamaica: Caribbean Challenge, Washington 1979; Girvan N., et al., The IMF and the Third World: the Case Study of Jamaica, 1974-1980, in: Development Dialogue, 2 (1980), S. $113 \mathrm{ff}$.

23 Angaben des WSLB Managements gegenüber der Verfasser. 
Da es im anglophonen Rechtskreis kein Universalbankensystem gibt, hat die WSLB das Hypotheken- und artverwandte Geschäft ausgegliedert und einem Tochterunternehmen, der 'Workers Savings and Loan Company', übertragen. ${ }^{24}$ Diese Gesellschaft hat sich besonders auf die Wohnbaufinanzierung spezialisiert. Sie ist ferner in den Bereichen Unternehmensfinanzierung, Vermögensverwaltung und Investment Fonds tätig. Die Trust Company ist mit ca. 25 \% Marktanteil das drittgrößte Spezialinstitut dieser Art auf Jamaika.

\section{Geschäftliche Entwicklung}

Die Arbeiterbank begann 1973 mit ca. J \$22 Mio. Einlagen, die sie von der Postsparkasse übernahm. Diese Spargelder waren in langfristigen Staatspapieren angelegt, so daß die WSLB diese Mittel nicht nach ihren Vorstellungen verwenden konnte. Während der stürmischen Anfangsentwicklung wuchs das Geschäftsvolumen schnell. ${ }^{25}$ Die Einlagen nahmen um 44,7\% von 24,1 Mio. (1973) auf 25 Mio. (1974) zu. Die Kreditvergabe stieg in diesem Zeitraum von 2,2 Mio. auf über 26 Mio. an.

Die Jahre 1975/76 brachten Jamaika hohe Inflationsraten, negatives Wirtschaftswachstum und zunehmende Zahlungsbilanzprobleme. ${ }^{26}$ Die labile ökonomische Situation wirkte sich auch auf den Bankensektor aus. Die Wachstumsraten schwächten sich deutlich ab. Die Arbeiterbank hatte unter großen Ausfällen zu leiden, da sich die Kreditsicherung und -überwachung als unzureichend erwies. Unregelmäßigkeiten führten schließlich zur Auswechselung des Spitzenmanagments. ${ }^{27}$ Der Marktanteil im K reditgeschäft war vorübergehend rückläufig. Die WSLB schloß die Geschäftsjahre 1975 und 1976 mit deutlichen Verlusten ab.

Trotz anhaltender Rezession unternahm die Arbeiterbank in den folgenden Jahren Anstrengungen, um den verlorenen Boden wieder aufzuholen. Im Geschäftsjahr 1977 nahmen die Depositen wieder um $39 \%$ und die Ausleihungen um $34 \%$ nominal zu, ohne daß sich die Ertragslage nachhaltig besserte. Wachstumsimpulse gingen u. a. von neu geknüpften Geschäftsbeziehungen mit dem 'National Housing Trustı und anderen parastaatlichen Organisationen aus. In den Jahren 1978 und 1979 schwächte sich die Wachstumsdynamik ab. Das ursprüngliche Ziel, bis 1981 einen Marktanteil von 30 \% zu erreichen, ${ }^{28}$ wurde weit verfehlt. Die Arbeiterbank konnte zu Beginn der achtziger Jahre lediglich einen Marktanteil von 8-9 \% erzielen. Die ambitionöse Zielsetzung spiegelte eher die Wunschvorstellungen eines auf Expansion bedachten Managements wieder, als daß sie eine realistische Einschätzung des Wachstumspotentials der Arbeiterbank lieferte.

24 Vgl. Walford, L. The Role of the Trust Company, Kingston 1977 (hekt.).

25 Vgl. zur geschäftlichen Entwicklung Tab. 2, Anhang.

26 Vgl. Girvan, N. et al., a.a.O., S. $121 \mathrm{ff}$.

27 Vgl. dazu folgende Presseberichte: The Workers Bank, Daily Gleaner 6./7./8. 3. 1975; Full Workers Bank Report on May 21, Daily Gleaner 18. 3. 1975.

$28 \mathrm{Vgl}$. A Study of Optimum Growth required for the WSLB, Kingston o. J., (hekt.), S. 1 f. 


\section{Einlagenpolitik}

Das Passivgeschäft der Arbeiterbank soll sich an folgenden Punkten orientieren:

- Entwicklung und Durchführung spezieller Sparprogramme;

- Förderung und Propagierung des Spargedankens insbesondere unter Arbeitern und Beziehern kleinerer Einkommen;

- Initiierung und Begünstigung von Sparprogrammen durch Organisationen wie Gewerkschaften, Credit Unions und Genossenschaften;

- Einführung spezieller Zwecksparprogramme für Ausbildungsmaßnahmen, Wohnungs- und Landkauf etc. ${ }^{29}$

Bisher hat die WSLB vor allem die Sparförderung in Arbeiterkreisen propagiert. Früher waren die Bezieher kleinerer Einkommen weitgehend vom Sparen in Geschäftsbanken ausgeschlossen, da diese Mindesteinlagen forderten. Eine solche Diskriminierung der Kleinsparer gibt es bei der Arbeiterbank nicht. In Verbindung mit den Tarifvertragsparteien hat die WSLB ein Vertragssparprogramm entwickelt, um der Arbeiterschaft eine attraktive Sparform anbieten zu können. Das Programm sieht vor, daß der vereinbarte Sparbetrag direkt vom Unternehmen an die WSLB überwiesen wird, wo er bis Ende der Vertragsdauer um $2 \%$ höher verzinst wird als normale Spareinlagen. Eine Teilnahme am Vertragssparen eröffnet weiterhin den Zugang zu günstigeren Kreditmöglichkeiten und erleichtert die Qualifizierung für eine Hypothekenzuteilung. Seit Einführung des Vertragssparens im Jahre 1974 ist die Zahl der beteiligten Unternehmen auf über 1400 gestiegen. Uber 10000 Arbeiter partizipieren an dieser Sparform, die sich allerdings nur für die Bezieher regelmäßiger Einkommen eignet. Gelegenheitsarbeiter und Arbeitslose können an dem Vertragssparprogramm nicht teilnehmen. Mehr sparpädagogische Zielsetzungen werden mit dem Schulsparen verfolgt, das die WSLB im Jamaika eingeführt hat. Ursprüngliche Pläne der Bank, in Kooperation mit den Credit Unions Zwecksparprogramme auf den Markt zu bringen, konnten nicht realisiert werden. Dieses Vorhaben scheiterte sowohl an persönlichen Rivalitäten zwischen WSLB Managern und Führern der Credit Union Bewegung als auch an der zunehmenden Zurückhaltung der Arbeiterbank, die sich von solchen Programmen keine Wachstumsimpulse versprach.

\section{Kreditpolitik}

Für das Aktivgeschäft der Arbeiterbank gelten folgende allgemeine Richtlinien:

- Präferierung von Jamaikanern und Klein- und Mittelbetrieben im Besitz jamaikanischer Staatsbürger;

- Ausrichtung auf die Kreditbedürfnisse von Arbeitern und Kleinbetrieben, insbesondere durch die Entwicklung neuer, auf die jamaikanischen Verhältnisse zugeschnittenen Kreditformen;

$29 \mathrm{Vgl}$. The Development of the Workers Bank, in: Workers Bank 4th Anniversary Supplement, Kingston 16. 10. 1977 , S. 2. 
- Unterstützung und Förderung von von Arbeitern gegründeten Betrieben und Selbsthilfeorganisationen;

- spezielle Förderung des Wohnungsbaus. ${ }^{30}$

In den ersten Jahren ihres Bestehens brachte die stark expansive Kreditpolitik der WSLB Rückschläge. Um die Wachstumsziele möglichst schnell erreichen zu können, wurden überwiegend mittlere und größere Kredite vergeben. ${ }^{31}$ Eine Reihe bedeutender Kredite wurde notleidend und belastete die Ertragslage der Bank. Fehlende Erfahrungen im Aktivgeschäft, die zu einer mangelhaften Absicherung und Uberwachung der Kredite führten, sowie ein Engagement im spekulativen Immobilienbereich, bewirkten die Verluste.

Das Kreditgeschäft konsolidierte sich erst allmählich. Bis 1977 nahmen die Anteile kleinerer Kredite an den Ausleihungen zu, so daß zu jenem Zeitpunkt die Kreditpolitik eher der Zielsetzung entsprach, Arbeiter und Kleinbetriebe mit Finanzmittel zu versorgen. Auch die Ausfallquoten besserten sich. 1977 wurden von den Personalkrediten bis J \$ 2000 ca. $4 \%$ und von denjenigen über $\mathrm{J} \$ 2001$ ca. $5 \%$ als 'nicht einholbar' eingestuft. ${ }^{32}$ Im Industriekreditgeschäft lag die Ausfallquote höher, allerdings hatte sie sich gegenüber 1974 deutlich gebessert.

Was die innovative Tätigkeit der Arbeiterbank angeht, so muß konstatiert werden, daß die WSLB weder neue Kreditformen auf den Markt gebracht hat noch im Bereich der Sicherheitsanforderungen neue Maßstäbe setzen konnte. Lediglich im Personalkreditgeschäft bemüht sich die Arbeiterbank, stärker als die übrigen Banken die individuelle Situation des Antragstellers zu berücksichtigen. Die im Vergleich recht günstige Entwicklung der Ausfallquoten in diesem Geschäftsbereich zeigt, daß die Arbeiterbank mit dieser flexibleren Haltung nicht schlecht gefahren ist.

Trotz anderslautender Absichtserklärungen hat die Arbeiterbank wenig für den Ausbau der Geschäftsbeziehungen zu den Gewerkschaften und Genossenschaften getan. Im Jahr 1978 machten die Kredite an diese Organisationen lediglich 1,4\% (Gewerkschaften) bzw. 1,1 \% (Genossenschaften) der gesamten Ausleihungen aus. Der Anteil der Genossenschaften am Aktivgeschäft erhöht sich allerdings, wenn die Kredite an den Zuckersektor berücksichtigt werden. Diese Gelder wurden allerdings nicht direkt an die Zukkerkooperativen ausgeliehen, sondern liefen über eine parastaatliche Förderungsorganisation, die für die Rückzahlung garantierte. Als Indiz dafür, daß es mit der sonstigen Zusammenarbeit mit den Genossenschaften und Credit Unions schlecht bestellt war, kann gelten, daß bis 1979 niemand in der WSLB Zentrale für die Kooperation mit Selbsthilfeorganisationen zuständig war. Die Arbeiterbank verfügte noch nicht einmal über ein Verzeichnis der auf Jamaika tätigen Genossenschaften und Credit Unions.

In der sektoralen Kreditverteilung unterscheidet sich die WSLB in Teilbereichen von

$30 \mathrm{Vgl}$. The Development of the Workers Bank, a.a.O., S. 2.

31 Vgl. Kirton, C., a.a.O., S. $321 \mathrm{f}$.

32 Angaben des WSLB Managements gegenüber dem Verfasser. 
den übrigen Geschäftsbanken. ${ }^{33}$ In den siebziger Jahren lagen die Ausleihungen an den Agrarsektor und die Industrie über dem Branchendurchschnitt. Unterdurchschnittlich waren dagegen die Kreditbeziehungen zum Handel, zu staatlichen Versorgungsunternehmen sowie nach den anfänglichen Rückschlägen zum Baugewerbe entwickelt. Das auffällig starke Engagement der Arbeiterbank im Agrarsektor ging allerdings nicht auf die freie Entscheidung der Bank zurück, diesen arbeitsintensiven Bereich mit Kreditmitteln zu versorgen. Ausschlaggebend war vielmehr das starke Interesse und auch der Druck der Manley Administration, zur Unterstützung der Agrarreformpolitik der Zitrus- und Zuckerwirtschaft neue Kredite zukommen zu lassen. Alle Agrarkredite waren staatsverbürgt und kein Ergebnis einer unternehmerischen Initiative der Arbeiterbank. Fürdas vergleichsweise stark entwickelte Industriekreditgeschäft waren ebenfalls einige Großkredite verantwortlich, die unter maßgeblicher Beteiligung der Regierung zustande kamen.

\section{Probleme}

Der Aufbau neuer Finanzierungsinstitutionen in post-kolonialen Gesellschaften ist nicht unproblematisch, wie das Beispiel Jamaika zeigt. ${ }^{34}$ Eine umfassende Beurteilung der Arbeiterbank kann nicht nur anhand der geschäftlichen Entwicklung erfolgen, sondern es müssen auch die gewachsenen historischen, gesellschaftlichen und psychologischen Strukturen mit bedacht werden. Eine problemorientierte Analyse muß daher folgende Gesichtspunkte mit berückșichtigen:

- die jahrhundertelange koloniale Abhängigkeit;

- den bis in die späten sechziger Jahre währenden Ausschluß Einheimischer von verantwortungsvollen Managementpositionen in den in Jamaika tätigen Tochterinstituten ausländischer Banken;

- das in der Bevölkerung aufgrund der kolonialen Erfahrung verbreitete Vertrauensdefizit gegenüber von Jamaikanern geführten kommerziellen Organisationen.

Vor diesem Hintergrund können die betrieblichen Probleme der WSLB grob als Management-, Kostenstruktur- und Imageprobleme klassifiziert werden. Ferner haben sich Schwierigkeiten bei der Umsetzung der bipolaren Unternehmenszielsetzung ergeben.

Die Arbeiterbank leidet unter einer hohen Personalfluktuation, insbesondere im Management. Seit ihrer Gründung hatte die Bank Probleme, qualifizierte Mitarbeiter zu verpflichten. Angestellte, die von anderen Banken kamen, haben häufig der Unternehmenszielsetzung der WSLB nicht ernst genommen und vielfach die Bank lediglich als Zwischenstation für eine Wirtschaftskarriere angesehen. Interne Aus- und Weiterbildungsprogramme konnten die Personalprobleme nicht nachhaltig entschärfen. Die Fluktua- 
tion hat sich negativ auf das Vertrauen der Kunden ausgewirkt und Zweifel bezüglich der zukünftigen Entwicklung der Bank in der Offentlichkeit geweckt.

Was die Kostenstruktur angeht, so erwies sich vor allem die Ubernahme der Postsparkasse als nicht unproblematisch. Die Úbernahme erbrachte der WSLB ein umfangreiches Sparkontenportfolio. Die Führung dieser durchweg kleinen Konten mit konventionellen Methoden erwies sich als sehr kostenintensiv. Dies gilt insbesondere für die ersten Jahre als die über 250000 aktiven und die ca. 200000 inaktiven Sparkonten manuell bearbeitet werden mußten. Die Situation wurde ferner-durch einen unerwarteten Anstieg der Sparzinsen erschwert, da die Postsparkasse die Spareinlagen langfristig in niedrig verzinslichen, unkündbaren Staatspapieren angelegt hatte. Ein weiteres Kostenstrukturproblem liegt in dem ungünstigen Depositmix. Im Vergleich zur Konkurrenz verfügt die Arbeiterbank nur über relativ wenige Termineinlagen und Girokonten. Auch fehlen günstige Refinanzierungsmöglichkeiten im Ausland.

Das Image der WSLB ist dadurch geprägt, daß die Arbeiterbank von weiten Bevölkerungskreisen als Regierungssparkasse angesehen wird. War dies in den ersten Geschäftsjahren durch die Ubernahme der Postsparkasse noch verständlich, so zeigt doch die weiterbestehende Assoziation der Arbeiterbank mit Regierungsstellen, daß es die Bank nicht verstanden hat, ein eigenes geschäftspolitisches Profil zu entwickeln. Die Beziehungen der Offentlichkeit zur Arbeiterbank waren zeitweise von Zurückhaltung und Verdächtigungen geprägt, wobei Management- und Ertragsprobleme die auslösenden Faktoren waren. ${ }^{35}$ Wiederholt wurde der Verdacht ausgesprochen, daß bei der Kreditvergabe Patronage und Begünstigung eine Rolle gespielt haben. ${ }^{36}$ Betroffene Regierungs- und Gewerkschaftsvertreter konnte diese Beschuldigungen nicht vollkommen entkräften.

Der Arbeiterbank ist es bisher nicht gelungen, ihre bipolare Zielsetzung adäquat umzusetzen. Wohl konnte die WSLB, die gegenwärtig einen Mittelplatz im jamaikanischen Bankgewerbe einnimmt, nachweisen, daß sie im Wettbewerb bestehen kann, aber ihre entwicklungs- und sozialpolitischen Ziele wurden weitgehend vernachlässigt. Gewerkschaftsvertreter im Board of Directors haben wiederholt auf dieses Mißverhältnis hingewiesen, ohne daß sie sich jedoch gegenüber dem auf rasche Expansion bedachten Management durchsetzen konnten. Die führenden WSLB Angestellten argumentieren, daß die Wettbewerbssituation keinen Raum für spezielle Programme und Initiativen lasse. Dem muß jedoch entgegen gehalten werden, daß es die Arbeiterbank bisher versäumt hat, ihren innovativen Spielraum zu nutzen. Obwohl mit der Gründung der WSLB die Hoffnung verbunden war, daß sie die als unbefriedigend empfundene Geschäftspolitik der übrigen Banken durch neue Spar- und Kreditformen und Dienstleistungen ergänzt, hat sich dies kaum bestätigt. Durch die weitgehende Orientierung am Größenwachstum und

35 Vgl. dazu die Presseberichterstattung, The Workers Bank, Daily Gleaner 6./7./8. 3. 1975; The Riddle of the Workers Bank, Sunday Gleaner 19. 2. 1978; Can the Workers Bank be saved?, Daily Gleaner 30. 4. 1978.

$36 \mathrm{Zu}$ den Anschuldigungen vgl. The Riddle of the Workers Bank, Daily Gleaner 7./8. 3. 1975; How the Workers Bank Exploits the Workers, in: Socialism/Kingston, 2 (1975), S. $36 \mathrm{ff}$. 
die Bewältigung der damit verbundenen Probleme konnte die WSLB kein eigenständiges geschäftspolitisches Profil entwickeln.

Im Bereich der Sparförderung wurden mit Ausnahme des Vertrags- und Schulsparens keine weiteren Initiativen mehr ergriffen. Dabei weist die vergleichsweise niedrige jamaikanische Sparquote noch auf Entwicklungsmöglichkeiten der Sparförderung hin. Beispiele aus Indien zeigen, daß selbst Sparprogramme, die auf Klein- und Kleinstsparbeträge abzielen, durchaus ökonomisch durchgeführt werden können. ${ }^{37}$ Auch im Kreditgeschäft hat sich die Arbeiterbank weitgehend an den übrigen Banken orientiert, ohne selbst innovativ tätig zu werden. Dabei demonstriert z. B. die Arbeiterbank von Trinidad, daß es durchaus möglich ist, mit Neuerungen im Aktivgeschäft sowohl Geld zu verdienen als auch wohnungs- und sozialpolitische Akzente zu setzen. ${ }^{38}$ Die WSLB hat es bisher versäumt, den dringend benötigten Beratungs- und Finanzierungsdienst für kleine gewerbliche und industrielle Kreditnehmer aufzubauen. Eine solche Dienstleistung hätte sicher mit geholfen, die Position der Arbeiterbank in diesem Marktbereich zu verbessern. Ferner wurden die Geschäftsbeziehungen zu dem genossenschaftlichen Sektor offensichtlich vernachlässigt. Die Credit Unions haben die Konsequenz gezogen und arbeiten inzwischen mit einer anderen Geschäftsbank zusammen. Auch auf gewerkschaftlicher Seite ist eine Entfremdung gegenüber der Arbeiterbank zu konstatieren. Befragte Gewerkschaftsführer und -mitglieder zeigten sich über die ihrer Meinung nach zu konservative Geschäftspolitik der WSLB enttäuscht.

Abschließend bleibt zu fragen, ob die Probleme der Arbeiterbank auf spezifisch jamaikanische Ursachen zurückzuführen oder ob sie nicht immanent im Arbeitermodell angelegt sind. Während einige betriebliche Schwierigkeiten in den wirtschaftlichen und institutionellen Verhältnissen der Karibikinsel begründet sind, deuten die Erfahrungen der übrigen lateinamerikanischen und karibischen Arbeiterbanken aber auch auf prinzipielle Probleme des Unternehmenstyps Arbeiterbank hin. ${ }^{39}$ In keinem Fall ist es einer Arbeiterbank gelungen, die in sie gesetzten Erwartungen zu erfüllen. Die Konkurrenzsituation mit anderen Banken erzwang ein marktkonformes Verhalten und ließ kaum einen Freiraum für eine konsequente Geschäftspolitik gemäß der bipolaren Unternehmenszielsetzung zu. Einige Arbeiterbanken konnten sich aus der staatlichen Umklammerung nicht lösen und fungieren heute quasi als staatliche Kreditinstitute (z. B. in Guatemala, Paraquay und Guyana). Andere Arbeiterbanken haben voll auf wirtschaftliche Expansion gesetzt und huldigen entwicklungs- und sozialpolitischen Zielen allenfalls noch in Sonntagsreden (z. B. in Venezuela). Wieder andere fristen ein Schattendasein, ohne die er-

37 Vgl. Bhatt, V. V., Innovations in Banking: The Syndicate's Experience, World Bank Domestic Finance Studies No. 46, Washington 1978; Mampilly, P., Innovations in Banking: The Indian Experience, World Bank Domestic Finance Studies No. 54, Washington 1979.

38 Die Bank hat ein erfolgreiches Konzept der dynamischen Baufinanzierung entwickelt, daß vor allem auf die Bezieher kleinerer und mittlerer Einkommen ausgerichtet ist. Vgl. Hackett, N. V., The Workers' Bank as an Intermediary in a Home Ownership Financing Market, in: American Institut of Free Labor Development (Hg.), a.a.O., S. $36 \mathrm{ff}$.

39 Vgl. dazu Mitchell, N., a.a.O., S. 184 ff.; Lewerenz, J., a.a.O., S. 48 ff. 
hofften Impulse für die Entwicklung eines gemeinwirtschaftlichen Sektors geben zu können (z. B. in Kolumbien und Honduras). Auch bei den übrigen Arbeiterbanken hat sich entweder ein dominierender Staatseinfluß durchgesetzt oder aber es ist dem Bankmanagement gelungen, sich weitgehend zu verselbständigen.

Vor diesem Erfahrungshintergrund müssen auch die Chancen der WSLB, die im Arbeiterbankgesetz niedergelegten Zielsetzungen voll zu verwirklichen, als gering eingeschätzt werden. Wahrscheinlicher ist, daß sich die Geschäftspolitik der jamaikanischen Arbeiterbank mehr und mehr der der übrigen Privatbanken angleichen und daß letztendlich von den reformpolitischen Absichten nichts übrig bleiben wird.

Anhang:

Tabelle 1

Eigenkapitalstruktur der WSLB (1979)

\begin{tabular}{|c|c|c|c|}
\hline Aktienklassen & $\begin{array}{l}\text { Genebuigtes } \\
\text { Kapital (in } \\
\text { '000 J } 8 \text { ) }\end{array}$ & $\begin{array}{l}\text { Eingezahltes } \\
\text { Kapital (in } \\
\text { ooo J } \$\end{array}$ & inox \\
\hline $\begin{array}{l}\text { A (Staat und parastaat- } \\
\text { licho Organisationen) }\end{array}$ & 1.000 & 500 & 12,4 \\
\hline B (Gewerikschaften) & 400 & 200 & 4,9 \\
\hline $\begin{array}{l}\text { C ( Offentlichkeit inkl. } \\
\text { Genossenscnaften und } \\
\text { Crodit Unıons) }\end{array}$ & 5.600 & 500 & 12,4 \\
\hline$D \underset{\text { Decht }}{(\text { Staat, }})$ kein Stimm- & 3.000 & 2.850 & 70,3 \\
\hline & 10.000 & 4.050 & 100,0 \\
\hline
\end{tabular}

Quelle: Workers Savings and Loan Bank, Annual Report 1979, 
Tabelle 2

Entwicklung der WSLB Einlagen und Kredite sowie der Marktanteile (1973-1979)

\begin{tabular}{|c|c|c|c|c|}
\hline Johr & $\begin{array}{l}\text { Einlagon } \\
(\text { in } ! 000 \mathrm{~J} \not)\end{array}$ & $\begin{array}{l}\text { Marktantell } \\
(\text { in } \%)\end{array}$ & $\begin{array}{l}\text { Kredite } \\
(\text { in } 1000 \mathrm{~J} g)\end{array}$ & $\begin{array}{l}\text { Marktanteil } \\
(\text { in } \mathscr{X})\end{array}$ \\
\hline 1973 & 24.153 & 4,6 & 2.273 & 0,5 \\
\hline 1974 & 34.964 & 5,9 & 26.095 & 4,9 \\
\hline 1975 & 47.926 & 6,6 & 28.760 & 4,5 \\
\hline 1976 & 53.442 & 6,9 & 35.952 & 5,2 \\
\hline 1977 & 74.227 & 7.9 & 48.495 & 7,5 \\
\hline 1978 & 84.924 & 8,2 & 57.341 & $7,6^{\circ}$ \\
\hline 1979 & 98.913 & 8,7 & 69.678 & 8,1 \\
\hline
\end{tabular}

Quelle: verschiedene Jahresberichte der WSLB 
ล Tabelle 3

Sektorale Kreditverteilung aller Geschäftsbanken (GB) und der Arbeiterbank (WSL

\begin{tabular}{|c|c|c|c|c|c|c|}
\hline & \multicolumn{2}{|c|}{1974} & \multicolumn{2}{|c|}{1975} & \multicolumn{2}{|c|}{1976} \\
\hline & OB & WSLB & GB & WSLB & GB & WSL \\
\hline 1. AgriculturefFishing & 4.6 & 4.3 & 10.2 & 19.6 & 11.7 & 28 \\
\hline 2. Mining & 1.1 & & 0.8 & & 0.9 & 0 \\
\hline 3. Manufacturing & 19.4 & 16.8 & 16.9 & $16 \cdot 0$ & $17.5 \cdot$ & 20.9 \\
\hline $\begin{array}{l}\text { 4. Construction and } \\
\text { Land Development }\end{array}$ & 21.6 & 36.2 & 20.3 & 33.0 & 18.1 & 18 \\
\hline 5. Financial Institutions & 3.1 & 3.7 & 3.1 & 0.0 & 2.8 & \\
\hline $\begin{array}{l}\text { 6. Transport, Storage and } \\
\text { Communication }\end{array}$ & ) & & ) & 7 & 4.0 & \\
\hline 7. Electricity, Gas, Water & $\int 4.7$ & $\int 2.0$ & $\int 5.1$ & 2.2 & 2.2 & \\
\hline 8. Goverment Services & 3.1 & 2.8 & 2.4 & 0.5 & 2.3 & \\
\hline 9. Distribution & 14.7 & 11.6 & 14.0 & 7.4 & 15.3 & \\
\hline 10. Tourism & 2.8 & ) & 1.8 & ) & 1.9 & \\
\hline 11. Entertainment & 0.8 & $\int 3.8$ & 0.6 & $\int 2.2$ & 0.6 & \\
\hline $\begin{array}{l}\text { 12. Professional and } \\
\text { other Services }\end{array}$ & 5.7 & 5.9 & 5.8 & 5.7 & 6.4 & \\
\hline 13. Personal & 18.4 & 11.5 & 19.0 & 13.3 & 16.3 & 11. \\
\hline 14. Oversaes Residents & 0.3 & - & 0.3 & - & 0.3 & \\
\hline
\end{tabular}

$(\mathrm{ccc)}$ E

Ouelle. Kreditstatistiken der WSLB Bank of Jamaica ( $\mathrm{Hg}$ ) Annual Reports, verschiedene Jahroänge 
bers, the differences between the richer and the poorer CARICOM-countries even increasing. Economic shortcomings as well as political difficulties were responsible for CARICOM's relative failure, the political difficulties being mostly engendered by the effects of the East-West-conflict in this part of the Third World.

This is why Guiana cannot dispense with its ties to Paris for the forseeable future. But the country will only benefit from them if the French government does accept the fact that by helping to build a more productive local economy and by transferring more political competences to the Guianese élite, it must prepare self-determination for the last continental overseas possession in Latin America, which cannot forever retain its present political status.

\section{The Development of the Jamaican Workers' Savings and Loan Bank in the Seventies}

\section{By Dieter Semmelroth}

In Jamaica there was growing criticism of the performance of foreign banks, which monopolized commercial banking in the early seventies. The reform - minded Prime Minister Michael Manley promoted the plan to establish a Jamaican - owned and run financial institution. In 1973 the Workers' Savings and Loan Bank (WSLB) was founded. The shares of this bank are held by major trade unions, co-operatives, the government and the public. The WSLB was inaugurated to function as a fully commercial banking institution with the aim of serving all segments of Jamaican society. Moreover it was expected that the WSLB should finance and develop programs and projects which promise high social and developmental return.

However, the overall performance of the WSLB during the seventies was rather disappointing. After having overcome some serious problems in its early years, the WSLB has managed in the meantime to achieve a middle position in the Jamaican banking business. But an evaluation of WLSB's savings and lending policy reveals that it does not differ significantly from that of its multinational competitors. The bank has largely failed to develop new and innovative projects and programs. Trade unions and co-operatives are increasingly dissatisfied by the "conservative" policy pursued by the WSLB management. Like other workers' banks in Latin America and the Caribbean, the Jamaican WSLB has not been able to meet the high expectations connected with its establishment. 